\title{
Thyroglobulin Measurement Through Fine-Needle Aspiration for Optimizing Neck Node Dissection in Papillary Thyroid Cancer
}

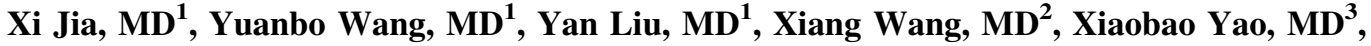 \\ Runyi Tao, BA ${ }^{4}$, Hui Liu, $\mathrm{MA}^{5}$, Aimin Yang, MD, $\mathrm{PhD}^{1}$, and Rui Gao, MD, $\mathrm{PhD}^{1}$ \\ ${ }^{1}$ Department of Nuclear Medicine, The First Affiliated Hospital of Xian Jiaotong University, Xi' an, Shaanxi, China; \\ ${ }^{2}$ Department of Ultrasound, The First Affiliated Hospital of Xi' an Jiaotong University, Xi'an, Shaanxi, China; ${ }^{3}$ Department \\ of Otorhinolaryngology - Head and Neck Surgery, The First Affiliated Hospital of Xi'an Jiaotong University, Xi'an, \\ Shaanxi, China; ${ }^{4}$ Department of Thoracic Surgery, The First Affiliated Hospital of Xi' an Jiaotong University, Xi' an, \\ Shaanxi, China; ${ }^{5}$ Biobank, The First Affiliated Hospital of Xi' an Jiaotong University, Xi' an, Shaanxi, China
}

\begin{abstract}
Background. Thyroglobulin measurement in fine-needle aspiration (FNA-Tg) is an additional diagnostic tool of lymph node metastasis (LNM) in papillary thyroid carcinoma (PTC). However, its performance as a preoperative indicator of lateral neck LNM in PTC is unclear. We evaluated the use of FNA cytology and FNA-Tg to detect neck LNM presurgery using a simple methodology, and established a cut-off value for diagnosing LNM in PTC.

Methods. We performed a retrospective cohort study based on hospital records, including 299 FNA-Tg measurements from 228 patients with PTC. The cut-off value for FNA-Tg was obtained through a receiver operating characteristic (ROC) curve analysis. The relationships between various parameters and FNA-Tg were analyzed using Spearman's correlation.

Results. Of 299 lymph nodes (LNs) from 228 patients following surgery, 151 were malignant and 148 were benign. The median FNA-Tg levels were $414.40 \mathrm{ng} / \mathrm{mL}$ and $6.36 \mathrm{ng} / \mathrm{mL}$ in the metastatic and benign LNs, respectively. An FNA-Tg cut-off value of $28.3 \mathrm{ng} / \mathrm{mL}$ had the best diagnostic performance $(93.38 \%$ sensitivity, $70.27 \%$ specificity, area under the ROC curve [AUC] 0.868 ) in the whole cohort. The diagnostic value performed
\end{abstract}

(C) The Author(s) 2021

First Received: 15 September 2020

Accepted: 14 July 2021;

Published Online: 12 August 2021

R. Gao, MD, PhD

e-mail: jacky_mg@xjtufh.edu.cn better in the lateral neck group (level II-V, $n=163$ ) than in the central neck group (level VI, $n=136$ ); in the lateral neck group, the sensitivity and specificity of the FNA-Tg cut-off $(16.8 \mathrm{ng} / \mathrm{mL})$ were $96.25 \%$ and $96.36 \%$, respectively.

Conclusions. FNA-Tg is a useful technique for the diagnosis of LNM before surgery, especially in lateral neck dissection.

Clinical trial registration number. ChiCTR1900028547.

Thyroid carcinoma is the most common endocrine malignancy. Papillary thyroid carcinoma (PTC), which is frequently associated with cervical lymph node metastasis (LNM), accounts for most thyroid carcinoma cases. ${ }^{1,2}$ The extent of the initial surgical approach relies on the accurate diagnosis of regional nodal metastases, especially lateral LNM (LLNM). ${ }^{3-5}$ Although extensive dissection could achieve a better diagnosis for LLNM, it has a higher potential for nerve injury and hemorrhage. Therefore, lateral compartment dissection is only recommended with fair evidence of LLNM, as per American Thyroid Association (ATA) guidelines. ${ }^{6}$

Less-invasive approaches, such as ultrasound (US)-guided fine-needle aspiration cytology (FNAC), are therefore commonly used. Unfortunately, false-negative (6-18\%) and non-diagnostic (up to 20\%) results are common, resulting in high rates of misdiagnosis. ${ }^{7-10}$ To improve the diagnostic accuracy of FNAC, thyroglobulin measurement through FNA (FNA-Tg) is frequently practiced in the preoperative diagnosis of LNM in PTC. In most studies, FNA-Tg provides additional diagnostic value for 
locoregional recurrence post-surgery, although its cut-off values are still controversial. ${ }^{11}$ To avoid missing metastatic disease in patients who may benefit from lymphadenectomy, a combination of FNAC and FNA-Tg has been applied to accurately differentiate LLNM from benign LNs; ${ }^{7}$ however, it is unclear whether FNA-Tg measurements could effectively indicate lateral neck LNM in PTC.

In the present study, we aimed to investigate the diagnostic value of FNA-Tg for the detection of LNM from PTC presurgery using a simple methodology, especially the lateral lymph nodes (LNs), and thus guide the necessity of lateral neck dissection.

\section{MATERIALS AND METHODS}

\section{Patients}

A total of 593 US-guided FNAs for cervical LNs were performed at The First Affiliated Hospital of Xi'an Jiaotong University in April-December 2019. A consecutive series of 445 patients with single or multiple suspicious cervical LNs underwent US-guided FNA to measure Tg levels (FNA-Tg). When multiple suspicious LNs were presented in one compartment, the representative nodes were selected for FNA. Only patients in whom PTC was confirmed by surgery were included in the study. Finally, a total of $299 \mathrm{LN}$ samples from 228 patients were investigated (Fig. 1).

We categorized dissected LNs as central (level VI) or lateral (level II-V) based on the American Joint Committee on Cancer classification (Eighth Edition). In total, 136 central LNs and 163 lateral LNs were included. The study was approved by the Hospital Ethics Committee of the First Hospital of Xi'an Jiaotong University (XJTU-ZD10) and registered in the Chinese Clinical Trial Register (registration number ChiCTR1900028547).

\section{Ultrasound and Ultrasound-Guided Fine-Needle Aspiration}

All 445 patients underwent US using a 10-12 MHz linear transducer prior to surgery, by experienced radiologists. Suspicious US characteristics included hyperechogenicity, cystic changes, calcification, abnormal vascularity, heterogeneous echogenicity, a round shape (longitudinal/transverse diameter ratio $<1.5$ ), and a loss of echogenic hilum. The locations (levels II-VI) of all cervical LNs were recorded based on the guidelines of the American Joint Committee on Cancer classification. ${ }^{7,12}$ When discordant results existed among different examiners, agreement was reached after a joint review and discussion of the data.

US-guided FNA was simultaneously performed using 22-gauge needles. Every suspicious LN was aspirated twice to guarantee enough samples for analysis. The needle was repeatedly inserted into the LN until sufficient material filled the needle hub. Immediately after the first aspirate was expelled onto glass, $5 \mu \mathrm{L}$ of the sample was added to $195 \mu \mathrm{L}$ of Tg-free serum. The clear supernatant after centrifugation was used for subsequent FNA-Tg measurement, and the remainder of the aspirate was smeared for cytological examination (FNAC). After smearing the sample from the second aspiration, the remainder of the material was rinsed in saline for processing as a cell block. All smears were immediately placed in $95 \%$ alcohol for Papanicolaou staining. We classified the cytologic specimens into three categories-malignant, benign, and

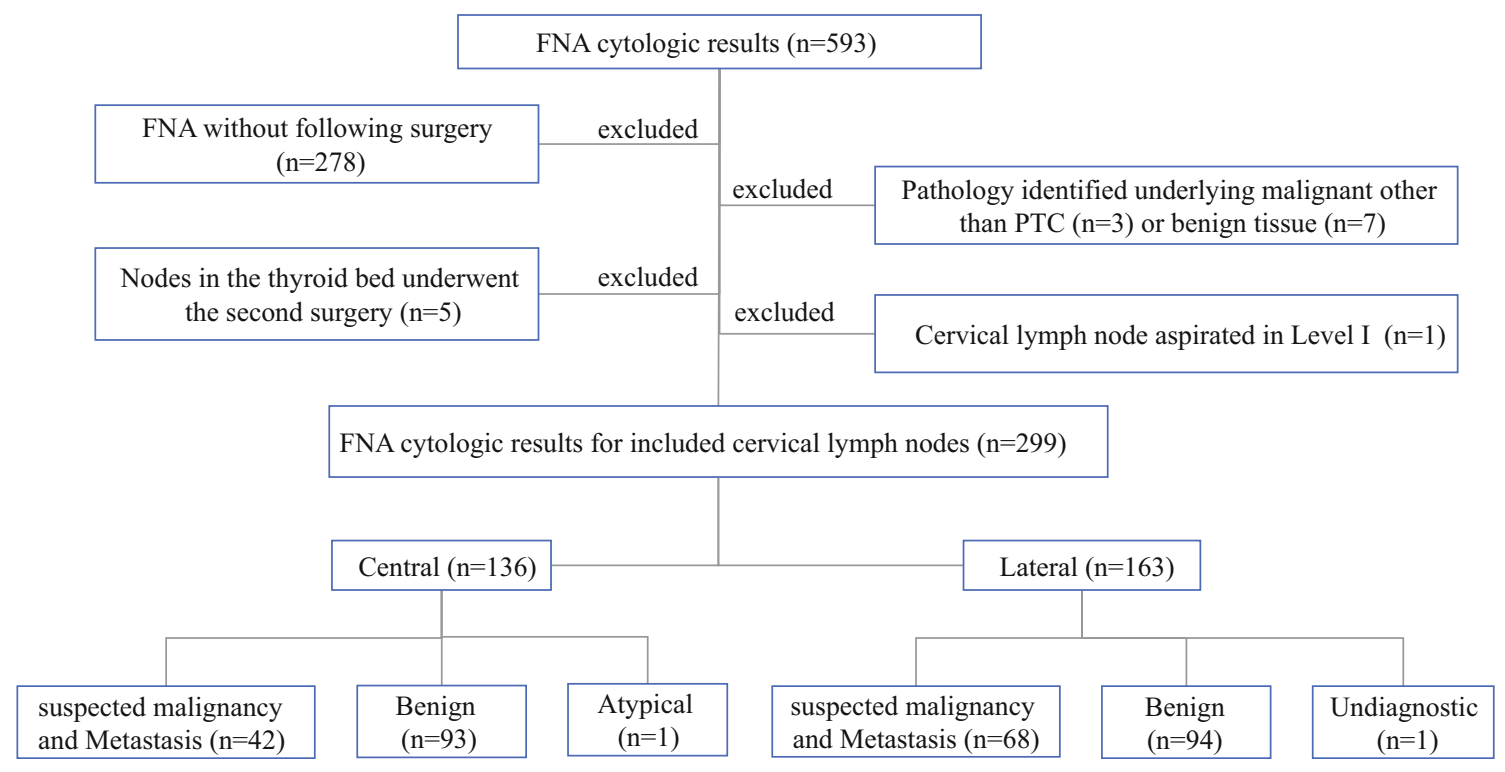

FIG. 1 Study selection and standard reference. FNA fine needle aspiration, PTC papillary thyroid carcinoma 
inadequate. If the cytology showed hemosiderin-laden macrophages, the specimen was classified as inadequate.

\section{Thyroglobulin Assessment}

Tg was assayed using a monoclonal antibody immunoradiometric assay (IRMA; CIS Bios International, Gif-surYvette, France). The functional sensitivity was $0.9 \mathrm{mg} / \mathrm{L}$, analytical sensitivity was $0.2 \mathrm{ng} / \mathrm{mL}$, and the interassay coefficient of variation (CV) was $6.9 \%$. Values exceeding the maximum $\mathrm{Tg}$ were recorded as $>500 \mathrm{ng} / \mathrm{mL}$. Serum $\mathrm{Tg}$ autoantibodies were measured using the Siemens IMMULITE 2000 (New York, NY, USA) assay (functional sensitivity, $40 \mathrm{kIU} / \mathrm{L}$; analytical sensitivity, $20 \mathrm{kIU} / \mathrm{L}$; and CV, 9\%).

\section{Surgical Protocol and Histopathological Analyses}

The extent of surgery was based on the results of FNAC + FNA-Tg. (1) When FNAC revealed malignant cells in the LNs, cervical neck dissection was performed along with total thyroidectomy. Cervical neck dissection involved central with/without unilateral/bilateral lateral compartment (levels II-V) dissection. ${ }^{13,14}$ (2) When the FNA-Tg levels were higher than $100 \mathrm{ng} / \mathrm{mL}$ in cases with negative cytological findings, neck dissection was also suggested in initial surgery. ${ }^{14,15}$ (3) In patients having LNs with suspicious US features but no definite metastatic cells found in FNAC and a level of FNA-Tg lower than $100 \mathrm{ng} /$ $\mathrm{mL}$, frozen section histologic analysis was only performed in cases required for it.

Final positive diagnoses required histological confirmation of metastatic thyroid carcinoma or clinical evidence of metastasis at follow-up, including an increased LN size observed by sonography and/or persistently increasing serum $\mathrm{Tg}$. The examination was undertaken at least twice yearly. Negative diagnoses were cases free of metastatic disease by the same criteria, based on a negative thyroidstimulating hormone (TSH)-stimulated Tg measurement or negative follow-up sonography. ${ }^{13}$

\section{Statistical Analyses}

Data following a normal distribution are expressed as mean \pm standard deviation (SD), and non-normally distributed data are expressed as median (interquartile range). The Chi-square test was used to compare counting data between groups, and measurement data were evaluated using Student's $t$-test or Mann-Whitney $U$ test. A receiver operating characteristic (ROC) curve analysis was used to determine the cut-off value of FNA-Tg for diagnosis of malignant LNs. The area under the ROC curve (AUC) and the confidence interval (CI) were also determined.
Spearman's correlation coefficients were used to estimate relationships between FNA-Tg and other parameters, while binary logistic regression was used to estimate multiple correlations between FNA-Tg results and other parameters. Using the pROC package in R, AUC, sensitivity, specificity, and $95 \%$ CIs were calculated under the negative binomial distribution. ROC curves were derived using the 'pROC' package in R (version 3.6.1; http://www.R-project. org). ${ }^{16,17}$ Other statistical analyses were performed in SPSS (version 24.0; IBM Corporation, Armonk, NY, USA). A $p$ value $<0.05$ was considered significant.

\section{RESULTS}

\section{Patient Characteristics}

The clinicopathologic characteristics of patients are summarized in Table 1 . This study included 165 women (72.37\%) and 63 men (27.63\%), and patient ages ranged from 20 to 81 years $(43.80 \pm 11.85$ years). Aspirated LNs were located at level II $(n=5)$, level III $(n=48)$, level IV $(n=101)$, level V $(n=9)$, and level VI $(n=136)$. Of the 228 patients who received thyroidectomy with neck dissection, 160 were given thyroidectomy with central LN dissection (CLND) only, 58 were given unilateral lateral LN dissection (LLND), and 10 were given bilateral LLND. A total of $265(88.63 \%)$ LNs were ipsilateral and 34 $(11.37 \%)$ were contralateral to the primary tumor. Metastatic LNs with bilateral cancers were considered ipsilateral LNs (27 patients with $36 \mathrm{LNs}$ ). Serum TSH and serum Tg levels did not differ between central LNs and lateral LNs ( $p=0.237$ and $p=0.295$, respectively). Serum $\operatorname{TgAb}$ was higher in central LNs than in lateral LNs $(p=0.001)$. It is worth noting that LN sizes in the central neck were significantly smaller than those in the lateral neck (median $6.00 \mathrm{~mm}$ vs. $9.00 \mathrm{~mm}, p=0.000$ ) (Table 2).

\section{Final Diagnosis}

In the final diagnosis, 151 (50.50\%) nodes were positive for metastatic disease and the remaining 148 (49.50\%) were negative. The positive nodes were determined by histopathology $(n=130)$, cytology $(n=13)$, and followup evidence $(n=8)$. The 148 negative nodes were determined by histological diagnosis $(n=74)$ and follow-up sonography $(n=74)$.

FNA-Tg was higher in malignant LNs than in benign LNs in the central group, lateral group, and the total samples. The median FNA-Tg values for the lateral group measured in metastatic and benign LNs were $498.45 \mathrm{ng} / \mathrm{mL}$ $(130.60,500 \mathrm{ng} / \mathrm{mL})$ and $0 \mathrm{ng} / \mathrm{mL}(0,3.40 \mathrm{ng} / \mathrm{mL})$, respectively. Other serum parameters revealed no 
TABLE 1 Patient characteristics

\begin{tabular}{|c|c|c|c|c|}
\hline \multirow{3}{*}{$\begin{array}{l}\text { Total patients } \\
\text { Total lymph nodes }\end{array}$} & \multicolumn{2}{|l|}{ Final outcome } & \multicolumn{2}{|l|}{ LND outcome } \\
\hline & 228 & & 158 & \\
\hline & 299 & & 205 & \\
\hline Age at diagnosis, years & $43.80 \pm 11.85$ & $0.000^{\mathrm{a}}$ & $41.67 \pm 11.60$ & $0.018^{\mathrm{a}}$ \\
\hline Groups, $<55$ years $/ \geq 55$ years & $185 / 43$ & $0.010^{\mathrm{b}}$ & $136 / 22$ & $0.229^{\mathrm{b}}$ \\
\hline Sex & & $0.009^{\mathrm{b}}$ & & $0.000^{\mathrm{b}}$ \\
\hline Male & $63(27.63 \%)$ & & $119(75.32 \%)$ & \\
\hline Female & $165(72.37 \%)$ & & $39(24.68 \%)$ & \\
\hline \multicolumn{5}{|l|}{ Primary tumor } \\
\hline BRAF & $181(79.39 \%)$ & $0.372^{\mathrm{b}}$ & $122(77.22 \%)$ & $0.884^{\mathrm{b}}$ \\
\hline Multifocality & $66(28.95 \%)$ & $0.170^{\mathrm{b}}$ & $43(27.22 \%)$ & $0.027^{\mathrm{a}}$ \\
\hline Hashimoto & $60(26.32 \%)$ & $0.092^{\mathrm{b}}$ & $56(35.44 \%)$ & $0.006^{\mathrm{b}}$ \\
\hline \multicolumn{5}{|l|}{ Lymph node } \\
\hline Location 1: Central/Lateral & $136 / 163$ & $0.289^{\mathrm{b}}$ & $131 / 74$ & $0.000^{\mathrm{b}}$ \\
\hline Location 2: levels $2 / 3 / 4 / 5 / 6$ & $5 / 48 / 101 / 9 / 136$ & $0.670^{\mathrm{c}}$ & $2 / 26 / 41 / 5 / 131$ & $0.000^{\mathrm{c}}$ \\
\hline Location 3: ipsilateral/contralateral & $265 / 34$ & $0.001^{\mathrm{b}}$ & $198 / 7$ & $0.238^{\mathrm{b}}$ \\
\hline
\end{tabular}

The follow-up results were only included in the final outcome, not in the LND outcome

LND lymph node dissection

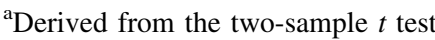

${ }^{b}$ Derived from the Chi-square test

${ }^{c}$ Derived from the Fisher's exact test
TABLE 2 Clinical parameters comparation

\begin{tabular}{lcll}
\hline & Central $[n=136]$ & Lateral $[n=163]$ & $p$-Value \\
\hline FNA-Tg & $156.75(33.95,500.00)$ & $15.00(0.00,500.00)$ & 0.000 \\
Serum-Tg & $9.71(1.00,19.25)$ & $11.50(1.31,25.35)$ & 0.295 \\
TgAb & $80.65(18.48,373.72)$ & $38.10(3.07,121.90)$ & 0.001 \\
TSH & $1.57(0.98,2.62)$ & $1.42(0.75,2.35)$ & 0.237 \\
LNDmax & $6.00(4.00,7.00)$ & $9.00(5.00,13.00)$ & 0.000
\end{tabular}

The follow-up results were only included in the final outcome, not in the LND outcome

FNA-Tg fine-needle aspirated thyroglobulin, LNDmax maximum diameter of lymph node, $L N D$ lymph node dissection, TSH thyroidstimulating hormone, $T g$ thyroglobulin, $T g A b$ thyroglobulin antibody

significant differences between malignant and benign LNs in the lateral group (Table 3).

\section{Cut-Off Values for Thyroglobulin Measurement in Fine-Needle Aspiration (FNA-Tg)}

In the lateral LNs $(n=163)$, an FNA-Tg cut-off of $16.80 \mathrm{ng} / \mathrm{mL}$ was the best diagnostic value, with $96.25 \%$ sensitivity and $96.39 \%$ specificity (AUC 0.967) (Fig. 2a). Negative and positive predictive values were $96.39 \%$ and $96.25 \%$, respectively. An FNA-Tg cut-off of $119.85 \mathrm{ng} / \mathrm{mL}$ had the best diagnostic performance in the central cervical LNs $(n=136)$, with sensitivity and specificity of $73.24 \%$ and $67.69 \%$, respectively (AUC $=0.725$ ) (Fig. 2b). When taken as a whole, $28.3 \mathrm{ng} / \mathrm{mL}$ was the best cut-off value, with a sensitivity and specificity of $93.38 \%$ and $70.27 \%$, respectively (AUC $=0.868)($ Fig. $2 \mathrm{c})$.

\section{Parameters Associated with FNA-Tg}

To evaluate the factors associated with FNA-Tg levels in cervical LNs, we examined the correlations between FNA-Tg levels and other clinical parameters. Interestingly, only the serum $\mathrm{TgAb}$ levels for central LNs correlated negatively with FNA-Tg levels (Spearman's correlation coefficient $=-0.232, p=0.028$ ), although the significant correlation of these two parameters was not observed in the lateral or whole groups. Serum Tg, TSH, or Hashimoto disease were not significantly correlated with FNA-Tg levels in all three groups (Table 4).

\section{Extent of Neck Dissection Based on Fine-Needle Aspiration Cytology and FNA-Tg}

Further analysis was performed to evaluate whether the FNA-Tg cut-off value for diagnosing LNM could optimize the extent of surgery. The results for total thyroidectomy with LN dissection (LND) are summarized in Table 5. 
TABLE 3 Comparison of parameters according to the different groups

\begin{tabular}{|c|c|c|c|}
\hline & Benign $[n=148]$ & Malignant $[n=151]$ & $p$-Value \\
\hline \multicolumn{4}{|l|}{$F N A-T g$} \\
\hline Whole & $6.36(0.00,47.02)$ & $414.40(118.20,500.00)$ & 0.000 \\
\hline Central & $49.40(23.50,257.40)$ & $322.40(91.20,500.00)$ & 0.000 \\
\hline Lateral & $0.00(0.00,3.40)$ & $498.45(130.60,500.00)$ & 0.000 \\
\hline \multicolumn{4}{|c|}{ Serum-Tg } \\
\hline Whole & $8.47(1.00,19.90)$ & $11.86(1.32,26.00)$ & 0.106 \\
\hline Central & $8.39(1.00,21.50)$ & $10.05(1.00,18.70)$ & 0.557 \\
\hline Lateral & $8.47(1.00,19.10)$ & $13.65(2.51,35.10)$ & 0.077 \\
\hline \multicolumn{4}{|c|}{ Serum- $T g A b$} \\
\hline Whole & $69.40(18.8,206.60)$ & $36.35(2.67,168.25)$ & 0.035 \\
\hline Central & $119.30(27.80,375.60)$ & $54.40(5.56,373.10)$ & 0.192 \\
\hline Lateral & $51.45(8.30,131.92)$ & $30.90(1.00,69.40)$ & 0.061 \\
\hline \multicolumn{4}{|l|}{ TPO } \\
\hline Whole & $1.00(1.00,822.00)$ & $1.00(1.00,592.00)$ & 0.399 \\
\hline Central & $124.40(1.00,1355.98)$ & $1.00(1.00,543.00)$ & 0.159 \\
\hline Lateral & $1.00(1.00,374.60)$ & $1.00(1.00,688.25)$ & 0.920 \\
\hline \multicolumn{4}{|l|}{$T S H$} \\
\hline Whole & $1.60(0.88,2.44)$ & $1.37(0.89,2.43)$ & 0.723 \\
\hline Central & $1.68(1.12,2.49)$ & $1.40(0.89,2.67)$ & 0.903 \\
\hline Lateral & $1.47(0.75,2.38)$ & $1.36(0.64,2.35)$ & 0.710 \\
\hline \multicolumn{4}{|c|}{ LNDmax } \\
\hline Whole & $7.00(4.00,11.00)$ & $6.00(3.75,10.25)$ & 0.345 \\
\hline Central & $6.00(4.25,7.00)$ & $5.00(1.40,7.00)$ & 0.120 \\
\hline Lateral & $9.00(4.00,13.00)$ & $9.00(6.00,14.25)$ & 0.629 \\
\hline
\end{tabular}

FNA-Tg fine-needle aspirated thyroglobulin, LNDmax maximum diameter of lymph node, LND lymph node dissection, TSH thyroid-stimulating hormone, $T g$ thyroglobulin, $T g A b$ thyroglobulin antibody, TPO Thyroid peroxidase (a)

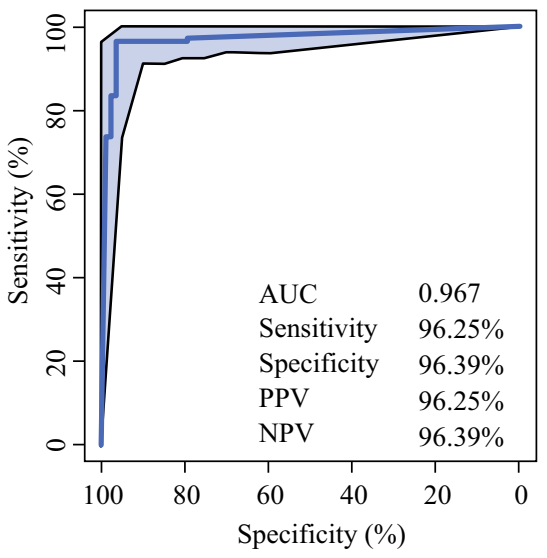

(b)

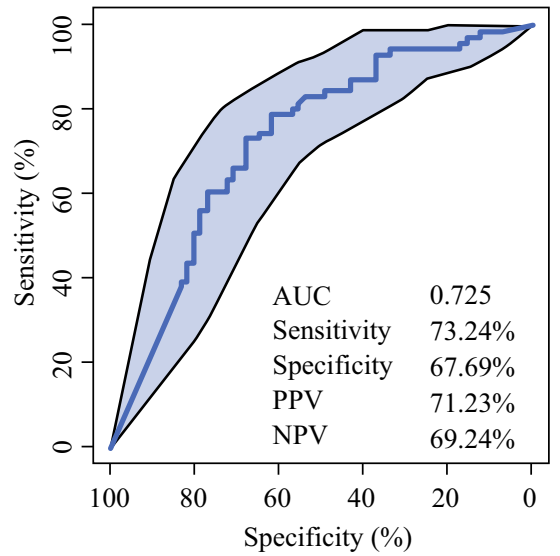

(c)

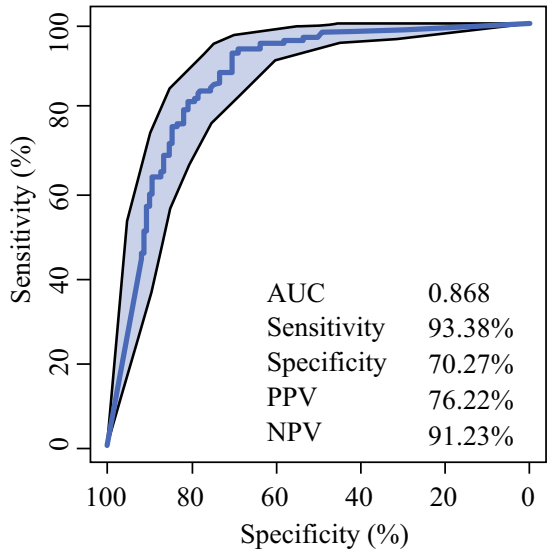

FIG. 2 Receiver operating characteristic curve of the three groups. a Lateral group. b Central group. c Whole group. $P P V$ positive predictive value, $N P V$ negative predictive value, $A U C$ area under the concentration-time curve.

Among 131 central neck LNs, 92 (70.23\%) demonstrated negative FNAC before surgery; however, postsurgical histopathological analyses revealed metastatic disease in 29 of these cases $(29 / 92,31.52 \%)$.
The diagnosis of FNA-Tg performed better in lateral neck LNs than in central neck LNs. In total, 74 LNs with clear results via FNAC were removed during lateral LND (LLND), 17 of which (22.97\%) showed FNAC-negative presurgery. In 5 of 17 LNs (29.41\%), metastatic disease 
TABLE 4 FNA-Tg correlation with the clinical test results

\begin{tabular}{llrrrr}
\hline & SerumTg & TgAb & TPO & TSH & Hashimoto \\
\hline Whole group & & & & & \\
Correlation coefficient & 0.079 & -0.086 & 0.009 & -0.025 & -0.011 \\
$\begin{array}{l}\text {-Value } \\
\text { Central group }\end{array}$ & 0.209 & 0.246 & 0.917 & 0.715 & 0.870 \\
Correlation coefficient & 0.109 & -0.232 & -0.088 & -0.081 & 0.113 \\
$p$-Value & 0.238 & 0.028 & 0.492 & 0.404 & 0.267 \\
Lateral group & & & & & \\
Correlation coefficient & 0.135 & -0.101 & -0.017 & 0.036 & -0.056 \\
$p$-Value & 0.121 & 0.330 & 0.885 & 0.706 & 0.541 \\
\hline
\end{tabular}

$\overline{F N A-T g}$ fine-needle aspirated thyroglobulin, $T S H$ thyroid-stimulating hormone, $T g$ thyroglobulin, $T g A b$ thyroglobulin antibody, TPO Thyroid peroxidase

\begin{tabular}{|c|c|c|c|c|}
\hline & \multicolumn{2}{|l|}{ FNAC- } & \multicolumn{2}{|l|}{ FNAC +} \\
\hline & Histology+ & Histology- & Histology+ & Histology- \\
\hline \multicolumn{5}{|c|}{ Central group } \\
\hline $\operatorname{Tg}+$ & 16 & 21 & 33 & 0 \\
\hline $\mathrm{Tg}-$ & 13 & 42 & 6 & 0 \\
\hline \multicolumn{5}{|c|}{ Lateral group } \\
\hline $\mathrm{Tg}+$ & 4 & $1^{\mathrm{a}}$ & 55 & 0 \\
\hline $\mathrm{Tg}-$ & 1 & 11 & 2 & 0 \\
\hline
\end{tabular}

$F N A-T g$ fine-needle aspirated thyroglobulin, $T g$ thyroglobulin, FNAC fine-needle aspiration cytology, $L N D$ lymph node dissection

${ }^{a}$ The inadequate cytology whose histology was negative was confirmed metastasis by 131-I imaging
TABLE 5 FNA-Tg diagnosis, cytology and histology with total thyroidectomy with LND are summarized the AUC of FNAC + FNA-Tg was higher than that of FNA-Tg alone (0.969 vs. 0.967), or that of FNAC alone $(0.969$ vs. 0.931$)$ in the lateral group, although with no significant differences ( $p=0.867$ and 0.063 , respectively). This suggested that using FNA-Tg alone without FNAC might be a choice in lateral LNs as it can offer comparable results at a much lower cost. ${ }^{7}$ The excellent negative predictive value could reduce the frequency of recytological examination, and the superior sensitivity could avoid the risk of reoperative complications, the extent of resection, and the cost of reoperation after the initial surgery.

In our study, the diagnostic accuracy of the whole group is consistent with that reported in previous research. ${ }^{20-24}$ Compared with the absolute value, the difference or ratio between FNA-Tg and serum $\mathrm{Tg}$ showed no diagnostic advantage (data not shown). However, the sensitivity and specificity values for the central group were much lower than their estimates for the overall group. LNs in the central region are more easily affected by blood contamination by circulating $\mathrm{Tg}$ at the time of sampling, or $\mathrm{Tg}$ leakage into the LN by lymph vessel draining. ${ }^{7,25,26}$ In the present study, only serum $\mathrm{TgAb}$ levels correlated negatively with FNA-Tg in the central LN group. It is quite possible that underestimation of the FNA-Tg concentration 
occurs, to some degree, in the presence of circulating $\operatorname{TgAb}$ in the central $\mathrm{LN}$ region but not in the lateral region. ${ }^{27}$ Consistent with correlation results, the number of $\mathrm{TgAb}$ positive cases with false negative results in the central group was significantly higher than in the lateral group $(p=0.023)$ [electronic supplementary table]. This might explain why LNs near the thyroid gland (e.g. paratracheal, pretracheal, and prelaryngeal nodes) before thyroidectomy yield inaccurate FNA-Tg values, irrespective of node pathology. ${ }^{28-30}$

FNA-Tg results are informative for LNs when the smallest diameter is $<10 \mathrm{~mm}$. FNA-Tg is particularly recommended in cases involving small, partially cystic LNs, as a significantly increased rate of non-diagnostic cytological results are indicated in these cases..$^{19,31,32}$ Our study highlighted the efficacy of FNA-Tg for small LNMs in the lateral neck. It is noteworthy that all nine LLNMs, not larger than $5 \mathrm{~mm}$, were successfully identified by FNA$\mathrm{Tg}$, supporting its routine use in preoperative planning to determine the extent of lateral neck dissection. Further large-scale and high-quality clinical trials are warranted to confirm its effectiveness.

There is wide debate regarding FNA-Tg cut-off values. The high variation among FNA procedures and sample preparation protocols contributes greatly to the different cutoff values used in different studies. ${ }^{33-41}$ It is not surprising that these differences result in substantial variation in the detection of cell density in specimens among studies, which could lead to the observation of a wide range of FNA-Tg levels in PTC LNM. ${ }^{41}$ In this study, we compensated for this variation in sample dilution by adopting a quantitative analytic method in which a fixed amount of the FNA aspirate $(5 \mu \mathrm{L})$ was diluted in Tg-free serum. The diagnostic value and best cut-off value were comparable with those reported in previous studies. ${ }^{20,33}$ Our standardized parameters, including the matrix type and volume of the aspirated sample, offer a potential standard operating procedure for further research, thereby enabling comparisons among studies.

Several limitations of the present study should be considered. First, the number of patients recruited was relatively small, and, additionally, other types of pathology other than PTC were not included in this study. FNA-Tg levels could be undetectable in some types of thyroid cancers, such as poorly differentiated thyroid cancer, ${ }^{42,43}$ which corresponds to the amount and intensity of $\mathrm{Tg}$ expression parallel with differentiation of the tumors. Furthermore, the number of metastatic disease cases, which was confirmed by postoperative histology, was relatively small, therefore future large-scale studies with longer follow-up periods are needed to verify our results. Second, we could not perform a node-by-node analysis for all LNs; instead, a level-by-level analysis was performed.
Additionally, the diagnosis of most benign lateral LNs was based on follow-up results. Despite these limitations, this study has important clinical implications. Our data confirmed that FNA-Tg is a reliable method for the recognition of metastatic disease in the lateral neck. Optimized lateral neck node dissection for patients with PTC might be performed based on an FNA-Tg cut-off of $16.8 \mathrm{ng} / \mathrm{mL}$. It is important to note that a patient whose cytology was inadequate, with an elevated FNA-Tg $(433.6 \mathrm{ng} / \mathrm{mL})$ in the lateral compartment, underwent the removal of $21 \mathrm{LNs}$ in the lateral neck and showed no metastatic lesions by postsurgery pathology. However, radioiodine-avid disease in the aspirated compartment was detected in the posttherapeutic whole-body scan. Accordingly, an FNA-Tg value of $>16.8 \mathrm{ng} / \mathrm{mL}$ in LLN might be a strong indicator for the existence of metastatic disease, even when it is not revealed by postsurgical analyses.

\section{CONCLUSION}

Diagnostic accuracies and cut-off values for FNA-Tg measurements differ between central and lateral cervical LNs. Regarding the diagnostic accuracy of lateral LNs, our standardized procedure was proved to be promising and should be considered to guide lateral neck dissection.

Supplementary Information The online version contains supplementary material available at https://doi.org/10.1245/s10434021-10549-2.

ACKNOWLEDGMENTS The authors thank Mr. Qi Fan and our colleagues for their helpful assistance.

FUNDING This study was supported by major new medical technology in the First Affiliated Hospital of Xian Jiaotong University (XJYFY-2019ZD10), the Key Point Research Project of Shaanxi Province (No. 2019SF-078, No. 2017SF-258), and the National Science Foundation of China (No. 81871389).

DISCLOSURES Xi Jia, Yuanbo Wang, Yan Liu, Xiang Wang, Xiaobao Yao, Runyi Tao, Hui Liu, Aimin Yang, and Rui Gao have no disclosures to declare.

OPEN ACCESS This article is licensed under a Creative Commons Attribution 4.0 International License, which permits use, sharing, adaptation, distribution and reproduction in any medium or format, as long as you give appropriate credit to the original author(s) and the source, provide a link to the Creative Commons licence, and indicate if changes were made. The images or other third party material in this article are included in the article's Creative Commons licence, unless indicated otherwise in a credit line to the material. If material is not included in the article's Creative Commons licence and your intended use is not permitted by statutory regulation or exceeds the permitted use, you will need to obtain permission directly from the copyright holder. To view a copy of this licence, visit http://creativecommons. org/licenses/by/4.0/. 


\section{REFERENCES}

1. Siegel RL, Miller KD, Jemal AJC. Cancer statistics, 2019. $C A$ Cancer J Clin. 2019;69(1):7-34.

2. Appetecchia M, Lauretta R, Barnabei A, et al. Epidemiology of simultaneous medullary and papillary thyroid carcinomas (MTC/ PTC): an Italian Multicenter Study. Cancers (Basel). 2019;11(10): 1516 .

3. Kim SK, Park I, Hur N, et al. Patterns, predictive factors, and prognostic impact of contralateral lateral lymph node metastasis in N1b papillary thyroid carcinoma. Ann Surg Oncol. 2017;24(7):1943-50.

4. Heng Y, Yang Z, Zhou L, et al. Risk stratification for lateral involvement in papillary thyroid carcinoma patients with central lymph node metastasis. Endocrine. 2020;68(2):320-8.

5. Park YM, Wang SG, Lee JC, et al. Metastatic lymph node status in the central compartment of papillary thyroid carcinoma: a prognostic factor of locoregional recurrence. Head Neck. 2016;38(Suppl 1):E1172-6.

6. Haugen BR, Alexander EK, Bible KC, et al. 2015 American Thyroid Association management guidelines for adult patients with thyroid nodules and differentiated thyroid cancer: the American Thyroid Association guidelines task force on thyroid nodules and differentiated thyroid cancer. Thyroid. 2016;26(1):1-133.

7. Al-Hilli Z, Strajina V, McKenzie TJ, et al. Thyroglobulin measurement in fine-needle aspiration improves the diagnosis of cervical lymph node metastases in papillary thyroid carcinoma. Ann Surg Oncol. 2017;24(3):739-44.

8. Hoda RS. Nondiagnostic/unsatisfactory thyroid fine needle aspiration on liquid-based preparations. Atlas of thyroid cytopathology on liquid-based preparations. New York: Springer; 2020. p. 27-34.

9. Zhu Y, Song Y, Xu G, et al. Causes of misdiagnoses by thyroid fine-needle aspiration cytology (FNAC): our experience and a systematic review. Diagn Pathol. 2020;15(1):1-8.

10. Koo DH, Song K, Kwon H, et al. Does tumor size influence the diagnostic accuracy of ultrasound-guided fine-needle aspiration cytology for thyroid nodules? Int $J$ Endocrinol. 2016;2016:3803647.

11. dos Santos Torres MR, Nobrega Neto SH, Rosas RJ, et al. Thyroglobulin in the washout fluid of lymph-node biopsy: what is its role in the follow-up of differentiated thyroid carcinoma? Thyroid. 2014;24(1):7-18.

12. Li Q, Zhao B, Lu J. Diagnostic value of fine-needle aspiration and thyroglobulin measurement in fine-needle aspiration for node metastasis in thyroid carcinoma before thyroidectomy. Chin J Ultrason Med. 2016;32(5):400-3.

13. Kim MJ, Kim E-K, Kim BM, et al. Thyroglobulin measurement in fine-needle aspirate washouts: the criteria for neck node dissection for patients with thyroid cancer. Clin Endocrinol. 2009;70(1):145-51.

14. Chung J, Kim EK, Lim H, et al. Optimal indication of thyroglobulin measurement in fine-needle aspiration for detecting lateral metastatic lymph nodes in patients with papillary thyroid carcinoma. Head Neck. 2014;36(6):795-801.

15. Lee JH, Lee HC, Yi HW, et al. Influence of thyroid gland status on the thyroglobulin cutoff level in washout fluid from cervical lymph nodes of patients with recurrent/metastatic papillary thyroid cancer. Head Neck. 2016;38:E1705-12.

16. Robin X, Turck N, Hainard A, et al. pROC: an open-source package for $\mathrm{R}$ and $\mathrm{S}$ plus to analyze and compare ROC curves. BMC Bioinform. 2011;12:77.
17. Kaforou M, Herberg JA, Wright VJ, et al. Diagnosis of bacterial infection using a 2-transcript host RNA signature in febrile infants 60 days or younger. JAMA. 2017;317(15):1577-8.

18. Khadra H, Mohamed H, Al-Qurayshi Z, et al. Superior detection of metastatic cystic lymphadenopathy in patients with papillary thyroid cancer by utilization of thyroglobulin washout. Head Neck. 2019;41(1):225-9.

19. Moon JH, Kim YI, Lim JA, et al. Thyroglobulin in washout fluid from lymph node fine-needle aspiration biopsy in papillary thyroid cancer: large-scale validation of the cutoff value to determine malignancy and evaluation of discrepant results. J Clin Endocrinol Metab. 2013;98(3):1061-8.

20. Pak K, Suh S, Hong H, et al. Diagnostic values of thyroglobulin measurement in fine-needle aspiration of lymph nodes in patients with thyroid cancer. Endocrine. 2015;49(1):70-7.

21. Sohn Y-M, Kim MJ, Kim E-K, et al. Diagnostic performance of thyroglobulin value in indeterminate range in fine needle aspiration washout fluid from lymph nodes of thyroid cancer. Yonsei Med J. 2012;53(1):126-31.

22. Frasoldati A, Toschi E, Zini M, et al. Role of thyroglobulin measurement in fine-needle aspiration biopsies of cervical lymph nodes in patients with differentiated thyroid cancer. Thyroid. 1999;9(2):105-11.

23. Li J, Zhang K, Liu X, et al. Cervical lymph node thyroglobulin measurement in washout of fine-needle aspirates for diagnosis of papillary thyroid cancer metastases. $\mathrm{Br} \quad \mathrm{J}$ Biomed Sci. 2016;73(2):79-83.

24. Eun NL, Son EJ, Kim J-A, et al. Comparison of the diagnostic performances of ultrasonography, CT and fine needle aspiration cytology for the prediction of lymph node metastasis in patients with lymph node dissection of papillary thyroid carcinoma: a retrospective cohort study. Int J Surg. 2018;51:145-50.

25. Weetman APMA, Wheeler MH, Hall R. Extrathyroidal sites of autoantibody synthesis in Graves' disease. Clin Exp Immunol. 1984;56(2):330-6.

26. Wang J, Jiang X, Xiao G, Zhou W, Hu Y. Excellent diagnostic performance of FNA-Tg in detecting lymph nodes metastases from papillary thyroid cancer. Future Oncol. 2020;16(33):2735-46.

27. Boi F, Baghino G, Atzeni F, et al. The diagnostic value for differentiated thyroid carcinoma metastases of thyroglobulin $(\mathrm{Tg})$ measurement in washout fluid from fine-needle aspiration biopsy of neck lymph nodes is maintained in the presence of circulating anti-Tg antibodies. J Clin Endocrinol Metab. 2006;91(4):1364-9.

28. Uruno T, Miyauchi A, Shimizu K, et al. Usefulness of thyroglobulin measurement in fine-needle aspiration biopsy specimens for diagnosing cervical lymph node metastasis in patients with papillary thyroid cancer. World $J$ Surg. 2005;29(4):483-5.

29. Sigstad E, Heilo A, Paus E, et al. The usefulness of detecting thyroglobulin in fine-needle aspirates from patients with neck lesions using a sensitive thyroglobulin assay. Diagn Cytopathol. 2007;35(12):761-7.

30. Achille G, Garrisi VM, Russo S, et al. Thyroglobulin determination in fine needle aspiration biopsy washout of suspicious lymph nodes in thyroid carcinoma follow up. Endocr Metab Immune. 2017;17(3):213-8.

31. Holmes BJ, Sokoll LJ, Li QK. Measurement of fine-needle aspiration thyroglobulin levels increases the detection of metastatic papillary thyroid carcinoma in cystic neck lesions. Cancer Cytopathol. 2014;122(7):521-6.

32. Lee YH, Seo HS, Suh S, et al. Cut-off value for needle washout thyroglobulin in athyrotropic patients. Laryngoscope. 2010;120(6):1120-4.

33. Grani G. Fumarola angela, thyroglobulin in lymph node fineneedle aspiration washout: a systematic review and meta-analysis 
of diagnostic accuracy. $J$ Clin Endocrinol Metab. 2014;99(6): 1970-82.

34. Sohn Y-M, Kwak JY, Kim E-K, et al. Diagnostic approach for evaluation of lymph node metastasis from thyroid cancer using ultrasound and fine-needle aspiration biopsy. Am J Roentgenol. 2010;194(1):38-43.

35. Wang $\mathrm{Y}$, Zhao $\mathrm{H}$, Wang $\mathrm{Y}-\mathrm{XJ}$, et al. Improvement in the detection of cystic metastatic papillary thyroid carcinoma by measurement of thyroglobulin in aspirated fluid. Biomed Res Int. 2016;2016:8905916.

36. Zhao H, Wang Y, Wang M-J, et al. Influence of presence/absence of thyroid gland on the cutoff value for thyroglobulin in lymphnode aspiration to detect metastatic papillary thyroid carcinoma. BMC Cancer. 2017;17(1):296.

37. Baldini E, Sorrenti S, Di Gioia C, et al. Cervical lymph node metastases from thyroid cancer: does thyroglobulin and calcitonin measurement in fine needle aspirates improve the diagnostic value of cytology? BMC Clin Pathol. 2013;13:7-7.

38. Pacini F, Fugazzola L, Lippi F, et al. Detection of thyroglobulin in fine needle aspirates of nonthyroidal neck masses-a clue to the diagnosis of metastatic differentiated thyroid-cancer. $J$ Clin Endocrinol Metab. 1992;74(6):1401-4.

39. Jeon SJ, Kim E, Park JS, et al. Diagnostic benefit of thyroglobulin measurement in fine-needle aspiration for diagnosing metastatic cervical lymph nodes from papillary thyroid cancer: correlations with US features. Korean J Radiol. 2009;10(2):106-11.

40. Lee MJ, Ross DS, Mueller PR, et al. Fine-needle biopsy of cervical lymph-nodes in patients with thyroid-cancer-a prospective comparison of cytopathologic and tissue marker analysis. Radiology. 1993;187(3):851-4.

41. Piotrkowski-Viale F, Reyes A, Dios A, et al. Effects of sample storage and diluents in the reliability of thyroglobulin measurement in the washout of fine needle aspirates. Endocrine. 2017;56(3):504-8.

42. Majstorov V, Gjorcheva D, Tripunoski T, et al. Thyroglobulin measurement in aspirates of neck lymph nodes in patients with benign and malignant thyroid disorders. Physioacta. 2015;9(3):27-35.

43. Borel A, Boizel R, Faure P, et al. Significance of low levels of thyroglobulin in fine needle aspirates from cervical lymph nodes of patients with a history of differentiated thyroid cancer. Eur $J$ Endocrinol. 2008;158(5):691-8.

Publisher's Note Springer Nature remains neutral with regard to jurisdictional claims in published maps and institutional affiliations. 